Document downloaded from:

http://hdl.handle.net/10251/139775

This paper must be cited as:

Polo, L.; Gómez-Cerezo, N.; Aznar, E.; Vivancos, J.; Sancenón Galarza, F.; Arcos, D.; Vallet, M.... (2017). Molecular gates in mesoporous bioactive glasses for the treatment of bone tumors and infection. Acta Biomaterialia. 50:114-126.

https://doi.org/10.1016/j.actbio.2016.12.025

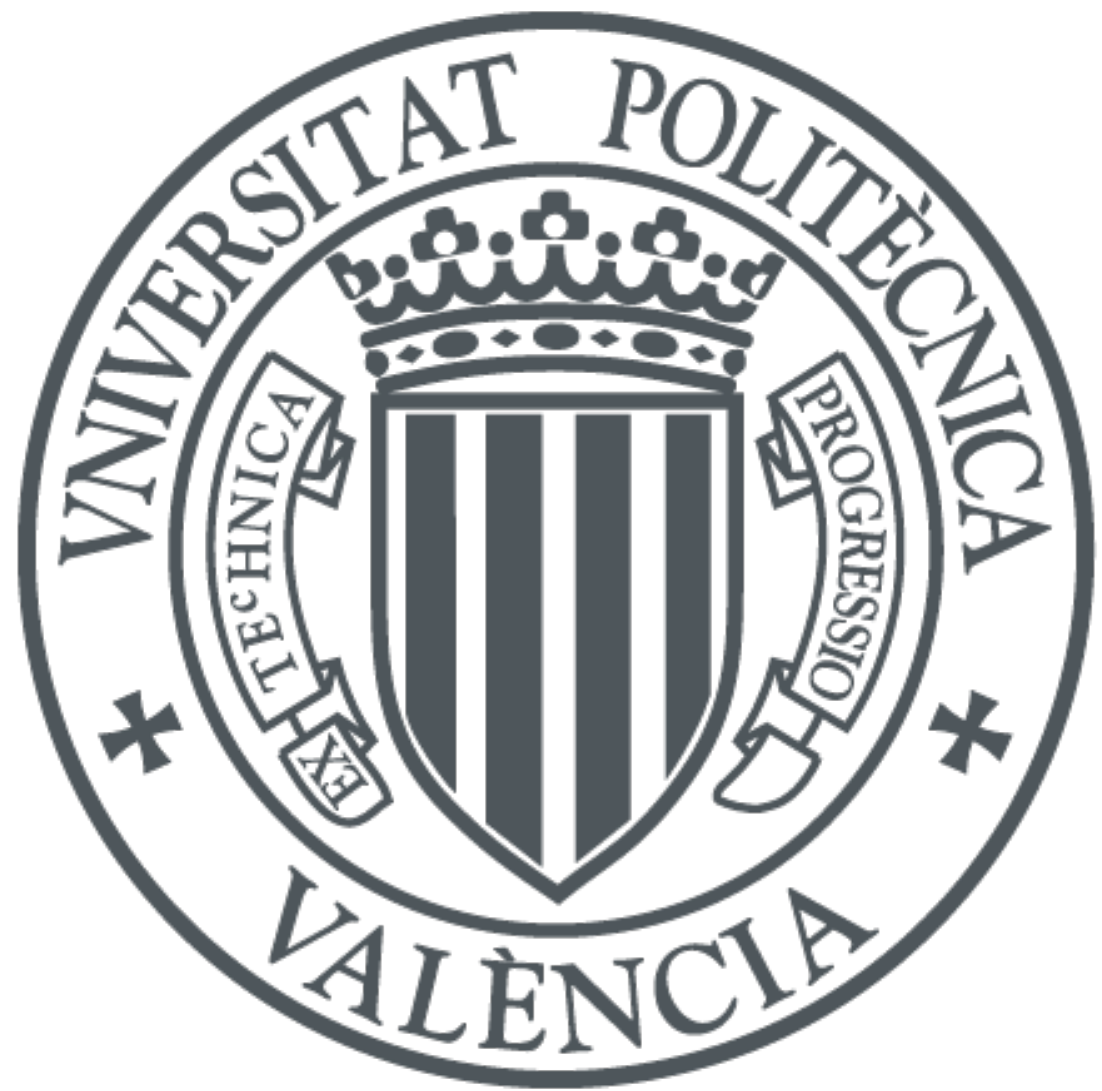

The final publication is available at

https://doi.org/10.1016/j.actbio.2016.12.025

Copyright Elsevier

Additional Information 


\section{Molecular gates in mesoporous bioactive glasses for the treatment of bone tumours and infection}

Lorena Polo, ${ }^{\mathrm{a}, \mathrm{c}, \ddagger}$ Natividad Gómez-Cerezo, ${ }^{\mathrm{b}, \mathrm{c}, \ddagger}$ Elena Aznar, ${ }^{\mathrm{a}, \mathrm{c}}$ José-Luis Vivancos, ${ }^{\mathrm{a}, \mathrm{c}}$

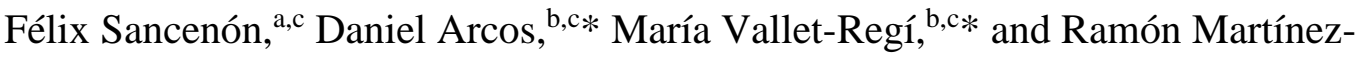
Máñez, ${ }^{\mathrm{a}, \mathrm{c} *}$

a Instituto Interuniversitario de Investigación de Reconocimiento Molecular y Desarrollo Tecnológico (IDM) Unidad Mixta Universitat de València-Universitat Politècnica de València, Camino de Vera s/n, 46022, Valencia, Spain

Phone: +34 963877343, Fax: +34 963879349.

b Departamento de Química Inorgánica y Bioinorgánica, Facultad de Farmacia, Universidad Complutense de Madrid Plaza Ramón y Cajal s/n 28040, Madrid, Spain

Phone: +34 913941843, Fax: +34 913941786

${ }^{\mathrm{c}}$ CIBER de Bioingeniería Biomateriales y Nanomedicina (CIBER-BBN), Spain

‡ These authors contributed equally to this work.

*Corresponding authors e-mail: rmaez@qim.upv.es, arcosd@ucm.es, vallet@ucm.es

Keywords: biomaterials, controlled release, gated mesoporous bioactive glasses.

\section{Abstract}

Silica mesoporous nanomaterials have been proved to have meaningful application in biotechnology and biomedicine. Particularly, mesoporous bioactive glasses are recently gaining importance thanks to their bone regenerative properties. Moreover, the mesoporous nature of these materials makes them suitable for drug delivery applications, opening new lines in the field of bone therapies. In this work, we have developed innovative nanodevices based on the implementation of adenosine triphosphate (ATP) and $\varepsilon$-poly-L-lysine molecular gates using a mesoporous bioglass as an inorganic support. The systems have been previously proved to work properly with a fluorescence probe and subsequently with an antibiotic (levofloxacin) and an antitumoral drug (doxorubicin). The bioactivity of the prepared materials has also been 
tested, giving promising results. Finally, in vitro cell culture studies have been carried out; demonstrating that this gated devices can provide useful approaches for bone cancer and bone infection treatments.

\section{Introduction}

Mesoporous bioactive glasses (MBGs) are a new generation of bioceramics designed for bone grafting and skeletal regenerative therapies [1,2]. These biomaterials exhibit the bone regenerative properties of bioactive glasses [3], but significantly increased due to their high surface area and porosity [4,5]. In addition, MBGs possess ordered mesoporous structures similar to those exhibited by pure silica mesoporous materials, which makes them excellent candidates as matrixes in drug delivery applications [6-8]. This synergy between osteogenic properties and local drug delivery capabilities is called to play a main role in field of skeletal therapies in near future. Several studies have been carried out to evaluate the behavior of MBGs as drug delivery systems [9], which have demonstrated that MBGs can release drugs following classical diffusion mechanism [10]. However, the real potential of MBGs remains still unknown. The possibility of supplying stimuli-responsive behavior to MBGs, in such a manner that they release the payload only when the pathological process occurs is unexplored.

From a different point of view, and in the context of on-command delivery, mesoporous silica has been used as an effective support for the development of controlled-release nanodevices because of their unique characteristics, such as high homogeneous porosity, inertness, robustness, thermal stability, and high loading capacity [11-14].

Consequently, a number of nanodevices for on-command delivery that can be triggered by target chemical [15-21], physical [22-26] or biochemical stimuli [27-34] have been designed recently. However these gated systems have been mainly developed in 
individual pure silica particles (usually nanometric) [35], and gated functionalities in bioactive compositions for bone regenerative purposes are practically unknown [36]. In order to explore new bone regeneration strategies, stimuli-responsive 3D macroporous scaffolds have been recently prepared by means of incorporating gated $\mathrm{SiO}_{2}$ mesoporous nanoparticles within a polymeric matrix [37]. However, as far as we are aware, none of these systems have been designed for combining bone regenerative properties and on-command drug delivery. The biological response of MBGs can be partially tailored by controlling the supramolecular mechanisms that rules the synthesis. For instance, we have recently demonstrated that the differentiation of pre-osteoblast toward osteoblast phenotype can be enhanced in contact with MBGs prepared with F68, a (EO) $)_{78}-(\mathrm{PO})_{30}-(\mathrm{EO})_{78}$ triblock copolymer which acts as a structure directing agent [38]. However, the complexity to incorporate the nanogates onto multicomponent $\mathrm{SiO}_{2}-$ $\mathrm{CaO}-\mathrm{P}_{2} \mathrm{O}_{5}$ systems has hindered perhaps the development of stimuli-responsive MBGs up to date. Nonetheless, the design of gated MBGs is highly appealing and might found a number of applications in advanced regenerative therapies.

Based on these concepts, it was in our aim to demonstrate the possibility of preparing stimuli-responsive MBGs, via the implementation of tailor-made gated ensembles on the surface of bioactive glasses. The final goal was to develop bioactive glasses able to regenerate bone tissue in bone defects while treating the causal pathology of such a defect, for instance bone infection or tumor extirpation. Therefore the mesopores of a selected MBG were capped with two different enzyme-responsive molecular gates based in the use of adenosine triphosphate (ATP) and $\varepsilon$-poly-L-lysine as caps (vide infra), which allowed controlled cargo release in the presence of alkaline phosphatase (ALP) and proteolytic enzymes, respectively. Proteolytic activity can be observed in the presence of different infectious pathogens as Escherichia coli $[39,40]$, whereas high 
levels of serum ALP have prognostic significance of osteosarcoma scenarios [41]. We envision that such tailor-designed systems may have potential applications for the treatment of bone tissue defects commonly associated to osteomyelitis and bone tumors extirpation.

\section{Materials and methods}

\subsection{Chemicals}

The chemicals tetraethyl orthosilicate (TEOS) (98\%), triethyl phosphate (TEP) (99\%), calcium nitrate $\mathrm{Ca}\left(\mathrm{NO}_{3}\right)_{2} \cdot 4 \mathrm{H}_{2} \mathrm{O}(99 \%)$, F68 (EO) ${ }_{78}-(\mathrm{PO})_{30}-(\mathrm{EO})_{78}$ triblock copolymer, tris(2,2'-bipyridyl)ruthenium(II) chloride hexahydrate ([Ru(bpy) $\left.\left.]_{3}\right]^{2+}\right)$, 3-[2-(2aminoethylamino)ethylamino]propyl-trimethoxysilane (N3), adenosine 5'-triphosphate disodium salt hydrate (ATP) (99\%), N-(3-Dimethylaminopropyl)-N'-ethylcarbodiimide hydrochloride (EDC), alkaline phosphatase (ALP) from bovine intestinal mucosa(buffered aqueous glycerol solution, $\geq 6,500$ DEA units/mg protein), 3(triethoxysilyl)propyl isocyanate, acetonitrile anhydrous (99.8\%), hydrochloric acid (37\%), levofloxacin (98\%), Dulbecco’s Modified Eagle’sMedium and pronase enzyme from S. griseus were purchased from Sigma-Aldrich Química S.A. Triethylamine (TEA) (98\%) was purchased from J.T. Baker Chemicals. $\varepsilon$-Poly-L-lysine was purchased from Chengdu Jinkai Biology Engineering Co., Ltd. Fetal Bovine Serum (FBS) was obtained from Gibco, BRL.LB medium was provided from Laboratorios Conda. Lglutamine, penicillin and streptomycin were purchased from BioWhittaker-VWR Europe. Doxorubicin used was obtained from the European Pharmacopoeia Reference Standard (Council of Europe EDQM).

\subsection{General Techniques}

FTIR spectroscopy was carried out with a Nicolet Magma IR 550 spectrometer. TEM images were obtained with a $100 \mathrm{kV}$ Jeol JEM-1010 microscope. SEM images were 
obtained with a 20kV JEOL F-6335 microscope. Differential thermal analysis was done in a TG/DTA Seiko SSC/5200 thermobalance between $50{ }^{\circ} \mathrm{C}$ and $1000{ }^{\circ} \mathrm{C}$ at a heating rate of $10^{\circ} \mathrm{C} \cdot \mathrm{min}^{-1}$. The textural properties of the calcined materials were determined by nitrogen adsorption porosimetry by using a Micromeritics ASAP 2020 porosimeter. To perform the $\mathrm{N}_{2}$ adsorption measurements, the samples were previously degassed under vacuum for 15 hours, at $150{ }^{\circ} \mathrm{C}$. The surface area was determined using the BrunauerEmmett-Teller (BET) method. The pore size distribution between 0.5 and $40 \mathrm{~nm}$ was determined from the adsorption branch of the isotherm by means of the Barret-JoynerHalenda (BJH) method. ${ }^{1} \mathrm{H}^{29} \mathrm{Si}$ and ${ }^{1} \mathrm{H}-{ }^{31} \mathrm{P} \mathrm{CP}$ (cross-polarization)/MAS (magic-anglespinning) and single-pulse (SP) solid-state nuclear magnetic resonance (NMR) measurements were performed to evaluate the different silicon and phosphorus environments in the synthesized samples. The NMR spectra were recorded on a Bruker Model Avance 400 spectrometer. Samples were spun at $10 \mathrm{kHz}$ for ${ }^{29} \mathrm{Si}$ and $6 \mathrm{kHz}$ in the case of ${ }^{31} \mathrm{P}$. Spectrometer frequencies were set to 79.49 and $161.97 \mathrm{MHz}$ for ${ }^{29} \mathrm{Si}$ and ${ }^{31} \mathrm{P}$, respectively. Chemical shift values were referenced to tetramethylsilane (TMS) and $\mathrm{H}_{3} \mathrm{PO}_{4}$ for ${ }^{29} \mathrm{Si}$ and ${ }^{31} \mathrm{P}$, respectively. The CP spectra were obtained using a proton enhanced CP method, at a contact time of 1 millisecond. The time period between successive accumulations was 5and 4 seconds for ${ }^{29} \mathrm{Si}$ and ${ }^{31} \mathrm{P}$, respectively, and the number of scans was 10000 for all spectra. Solid-state ${ }^{13} \mathrm{C}$ spectra were obtained with a Bruker Model Avance 400 spectrometer 75.46 MHz. For the cell proliferation test, the absorbance was measured using a Helios Zeta UV-VIS spectrophotometer.

\subsection{Synthesis of mesoporous bioactive glasses functionalized}

\subsubsection{Synthesis of the mesoporous bioactive glass (S1)}

$85 \% \mathrm{SiO}_{2}-10 \% \mathrm{CaO}-5 \% \mathrm{P}_{2} \mathrm{O}_{5}$ (\% mol) mesoporous glass (S1) was synthetized by evaporation induced self-assembly (EISA) method, using F68 (EO) ${ }_{78}-(\mathrm{PO})_{30}-(\mathrm{EO})_{78}$ 
triblock copolymer as structure directing agent. TEOS, TEP and calcium nitrate $\mathrm{Ca}\left(\mathrm{NO}_{3}\right)_{2} \cdot 4 \mathrm{H}_{2} \mathrm{O}$ were used as $\mathrm{SiO}_{2}, \mathrm{P}_{2} \mathrm{O}_{5}$ and $\mathrm{CaO}$ sources, respectively. In a typical synthesis, $2 \mathrm{~g}$ of F68 were dissolved in $30 \mathrm{~g}$ of ethanol with $0.5 \mathrm{ml}$ of $\mathrm{HCl} 0.5 \mathrm{M}$ solution at room temperature. Afterward, $3.70 \mathrm{~g}$ of TEOS, $0.34 \mathrm{~g}$ of TEP and $0.49 \mathrm{~g}$ of $\mathrm{Ca}\left(\mathrm{NO}_{3}\right)_{2} \cdot 4 \mathrm{H}_{2} \mathrm{O}$ were added under stirring in 3 hours intervals. The resulting solution was stirred during 12 hours and casted into Petri dishes (9 cm in diameter). The colorless solution was evaporated at $30{ }^{\circ} \mathrm{C}$ during 11 days. Eventually, the dried gels were removed as homogeneous and transparent membranes, and heated at $700{ }^{\circ} \mathrm{C}$ for 3 hours under air atmosphere. Finally the MBG powder was gently milled and sieved, collecting the particle size fraction below $40 \mu \mathrm{m}$.

\subsubsection{Synthesis of S2}

$1 \mathrm{~g}$ of $\mathbf{S 1}$ was suspended in $30 \mathrm{~mL}$ of anhydrous acetonitrile under inert atmosphere. Then, $1 \mathrm{~mL}$ (3.88 mmol) of 3-[2-(2-aminoethylamino)ethylamino]propyltrimethoxysilane (N3) was added and the mixture was stirred for 5.5 hours. Finally, the solid was filtered, washed with $\mathrm{H}_{2} \mathrm{O}$ repeatedly and dried under vacuum for 12 hours.

\subsubsection{Synthesis of S3}

$200 \mathrm{mg}$ of solid S2 were suspended in a solution of EDC $0.1 \mathrm{M}$ and ATP 1.8 M, previously adjusted to $\mathrm{pH} 7$ with $\mathrm{NaOH}$. The suspension was stirred for 6 hours at room temperature. The resulting solid was filtered and dried under vacuum for 12 hours.

\subsubsection{Synthesis of S4}

In a typical synthesis, $1 \mathrm{~g}$ of $\mathbf{S 1}$ was suspended in $50 \mathrm{~mL}$ of methanol, inside a roundbottom flask under inert atmosphere. Then, an excess of 3(triethoxysilyl)propylisocyanate (isocyanate) $(1 \mathrm{~mL}, 4 \mathrm{mmol})$ was added and the final mixture was stirred for 5.5 hours at room temperature. The solid was filtered, washed with $\mathrm{H}_{2} \mathrm{O}$ repeatedly, and dried under vacuum for 12 hours. 


\subsubsection{Synthesis of S5}

A solution of $1 \mathrm{~g}$ of $\varepsilon$-poly-L-lysine $(0.2 \mathrm{mmol})$ and TEA $(1.6 \mathrm{~mL}, 11.5 \mathrm{mmol})$ in $20 \mathrm{~mL}$ of methanol was added onto the solid S4, and the mixture was stirred for 2 hours. Finally, the solid was filtered off and dried under vacuum.

\subsubsection{Synthesis of S3-Ru and S5-Ru}

In order to carry out the proof of concept about the functioning of ATP and $\varepsilon$-poly-llysine based gates, both systems were loaded with a fluorescence dye. For this purpose $2 \mathrm{~g}$ of S1 were suspended in a solution of $1.6 \mathrm{~g}$ tris(2,2'-bipyridil)ruthenium(II) chloride hexahydrate ([Ru(bpy) $\left.)_{3}\right]^{2+}$ dye in $70 \mathrm{~mL}$ of anhydrous acetonitrile in a round-bottomed flask. Then, $10 \mathrm{~mL}$ of acetonitrile were distilled with a dean-stark, in order to remove the possible water present in the pores of the solid. Afterwards, the mixture was stirred at room temperature during 24 hours, in order to achieve maximum loading in the pores of the MBG scaffolding. The resulting solid was then filtered and dried under vacuum for 12 hours. Subsequently, the solid was functionalized and capped as S3 and S5 respectively, as described above. Once dried, the solids were suspended in $5 \mathrm{~mL}$ of water and stirred in short washing, in order to remove the excess of dye remaining on the surface of the materials. The solids were filtered again and dried under vacuum for 12 hours.

\subsubsection{Synthesis of S3-Levo and S5-Levo}

In order to test the functioning of ATP and $\varepsilon$-poly-L-lysine based gates as on command antibiotic delivery devices, both systems were loaded with levofloxacin. For this purpose $800 \mathrm{mg}$ of $\mathbf{S 1}$ were suspended in a solution of $400 \mathrm{mg}(1.11 \mathrm{mmol})$ of levofloxacin in $20 \mathrm{~mL}$ of methanol inside a round-bottom flask under inert atmosphere. The mixture was stirred at room temperature during 24 hours to achieve maximum loading in the pores of the MBG. Subsequently, the solution was split in two and each 
part was functionalized in a similar way to S3 and S5 respectively. Finally S3-Levo and S5-Levo were washed in water to remove the excess of antibiotic remaining on the surface.

\subsubsection{Synthesis of S3-Dox}

In order to test the functioning of the ATP based gate as on command antitumoral delivery devices, $30 \mathrm{mg}$ of solid $\mathbf{S} 2$ and $6 \mathrm{mg}$ of doxorubicin were suspended in $5 \mathrm{~mL}$ of methanol and stirred for 50 hours. Then, the solid was centrifuged and dried under vacuum for 1 hour. Finally the solid was introduced in a solution of EDC $0.1 \mathrm{M}$ and ATP $1.8 \mathrm{M}$ previously adjusted to $\mathrm{pH} 7$ with $\mathrm{NaOH}$. The suspension was stirred for 6 hours at room temperature. The resulting solid was centrifuged, washed several times with water and methanol and dried under vacuum for 12 hours.

Table 1 contains a summary of the synthesized solids.

\subsection{Stimuli-responsive studies}

\subsubsection{Stimuli-responsive studies with S3-Ru and S5-Ru}

$4 \mathrm{mg}$ of the S3-Ru and S5-Ru were suspended in $10 \mathrm{~mL}$ of water, and the $\mathrm{pH}$ was adjusted to 7.6 with $\mathrm{NaOH}$. Both suspensions were split in two in order to study the dye release in the absence or presence of the corresponding stimuli, i.e. ALP and pronase for S3-Ru and S5-Ru, respectively. The two samples were stirred at $400 \mathrm{rpm}$ and $37^{\circ} \mathrm{C}$, and then several $250 \mu \mathrm{L}$ aliquots were taken for each sample at different times. These aliquots were filtered with PFTE filters $(0.22 \mu \mathrm{m})$ to monitor the $\left[\mathrm{Ru}(\mathrm{bpy})_{3}\right]^{2+}$ release ( $\lambda_{\text {ex }} 454 \mathrm{~nm}, \lambda_{\text {em }} 593 \mathrm{~nm}$ ) by fluorescence spectroscopy

\subsubsection{Stimuli-responsive studies with S5-Levo}

$4 \mathrm{mg}$ of S5-Levo were suspended in $10 \mathrm{~mL}$ of water, and the $\mathrm{pH}$ was adjusted to 7.6 with $\mathrm{NaOH}$. The suspension was split in two in order to study the levofloxacin release in the absence and the presence of E.coli (final concentration $10^{5}$ cells $\mathrm{mL}^{-1}$ ). The two 
samples were stirred at $400 \mathrm{rpm}$ and $37^{\circ} \mathrm{C}$, and then several $250 \mu \mathrm{L}$ aliquots were taken for each sample at different times. These aliquots were filtered with PFTE filters $(0.22$ $\mu \mathrm{m})$ to monitor the levofloxacin release $\left(\lambda_{\mathrm{ex}} 292 \mathrm{~nm}, \lambda_{\mathrm{em}} 494 \mathrm{~nm}\right)$ by fluorescence spectroscopy.

\subsubsection{Stimuli-responsive studies with S3-Dox}

$4.6 \mathrm{mg}$ of S3-Dox were suspended in $2 \mathrm{~mL}$ of $\mathrm{H}_{2} \mathrm{O} \mathrm{pH}$ 7.6. Then, $0.5 \mathrm{~mL}$ of this suspension was placed on a Transwell permeable support with polycarbonate membrane $(0.4 \mu \mathrm{m})$. The well was filled with $1.5 \mathrm{~mL}$ of $\mathrm{H}_{2} \mathrm{O} \mathrm{pH} 7.6$ and the suspension was stirred at $37^{\circ} \mathrm{C}$ and $100 \mathrm{rpm}$ during all the experiment. To study the enzymatic responsiveness of the solid, the suspension was in contact with ALP on a Transwell. The amount of cargo released was determined by fluorescence spectrometry $\lambda_{\text {exc }} 490, \lambda_{\text {em }} 514 \mathrm{~nm}$, and the solution outside the Transwell insert was replaced with fresh medium, with or without ALP.

\subsection{Bioactivity assays with solids S3 and S5}

Assessments of in vitro bioactivity were carried out on S3 and S5 solids. For this purpose, $40 \mathrm{mg}$ of the solids were soaked into $6 \mathrm{~mL}$ of filtered simulated body fluid (SBF) [46] in polyethylene containers at $37^{\circ} \mathrm{C}$ under sterile conditions [38]. The evolution of the solids surfaces were analysed by Fourier transform infrared (FTIR) spectroscopy and scanning electron microscopy (SEM).

\subsection{Biological assessment of the gated materials}

\subsubsection{E. coli DH5 $\alpha$ culture conditions}

For viability studies, bacteria Escherichia coli (E. coli), cell culture DH5 $\alpha$ was used. Bacteria cells were maintained in glycerol $15 \%$ at $-80{ }^{\circ} \mathrm{C}$. For the assays, cells were grown for 24 hours at $37^{\circ} \mathrm{C}$ and under constant stirring with $5 \mathrm{~mL}$ of LB medium. Cells 
from $1 \mathrm{~mL}$ culture were collected by centrifugation for 30 seconds at $13000 \mathrm{rpm}$ and resuspended in $1 \mathrm{~mL}$ of milliQ water at $\mathrm{pH}$ 7.6. Then a dilution of $2 \cdot 10^{4}$ cells $\cdot \mathrm{mL}^{-1}$ was prepared, in order to achieve a final concentration of $10^{4}$ cells $\cdot \mathrm{mL}^{-1}$. The same procedure was carried out for both kinetic and viability assays.

\subsubsection{Clonogenic cell viability assay with S5-Levo, levofloxacin and \&-poly-L-lysine}

For the clonogenic cell viability assay with S5-Levo, different suspensions of S5-Levo containing bacteria (final concentration $10^{4}$ cells $\cdot \mathrm{mL}^{-1}$ ) were prepared, achieving the final solid concentrations of 800, 500, 300, 200, 160, 120, 80, 60, 40, 20, 10, 5, 1 and 0

$\mu \mathrm{g}$ solid $\cdot \mathrm{mL}^{-1}$. The samples were stirred at $180 \mathrm{rpm}\left(37^{\circ} \mathrm{C}\right)$ during 10 minutes. Then, the suspensions were suitably diluted with milliQ water (pH 7.6) in order to obtain a cell growth easy to quantify. Finally, $100 \mu \mathrm{L}$ of the new dilutions were seeded in LB plates (3\% agar) and incubated at $37^{\circ} \mathrm{C}$ for 24 hours. Then, Colony Formation Units (CFU) were quantified.

To test the cytotoxicity of the non-loaded $\varepsilon$-poly-L-lysine capped solid (S5), the same experiment was carried out with final solid concentratons of 600, 300, 200, 100, 50, 30, 20, 5 and $0 \mu \mathrm{g}$ solid $\cdot \mathrm{mL}^{-1}$.

To determine the cytotoxicity of free levofloxacin and free $\varepsilon$-poly-L-lysine, final concentrations of 2000,1000, 800, 600, 400, 300, 250, 200, 150, 100, 60, 30, 10 and 0 $\mathrm{ng} \cdot \mathrm{mL}^{-1}$ (levofloxacin) and 600, 400, 200, 100, 80, 50, 40, 30, 10 and $0 \mathrm{ng} \cdot \mathrm{mL}^{-1}(\varepsilon-$ poly-L-lysine) were achieved. In order to test the specificity of the gates, solid S3-Levo (capped with ATP) was submitted to the same procedure.

\subsubsection{Human Osteosarcoma (HOS) cells culture test}

HOS cells were seeded on well culture plates (CULTEK), at a density of 40000 cell per mL in Dulbecco's Modified Eagle’s Medium with 10\% foetal bovine serum, 1 mM Lglutamine, penicillin $\left(200 \mathrm{mg} \cdot \mathrm{mL}^{-1}\right)$, and streptomycin $\left(200 \mathrm{mg} \cdot \mathrm{mL}^{-1}\right)$, under a $\mathrm{CO}_{2}$ 
(5\%) atmosphere at $37^{\circ} \mathrm{C}, 24$ hours to reach the confluence in each cell plate.

Thereafter, S3-Dox solid was added on the seed cells at half confluence at $200 \mu \mathrm{g} \cdot \mathrm{mL}^{-}$

${ }^{1}$ concentration.In order to study the stimuli-responsive behaviour of the ATP gate, ALP was added to the culture wells to simulate a scenario of ALP activity excess.

\subsubsection{Cell proliferation test}

Cell proliferation in contact with S3 and S3-Dox was determined by the MTT method. Samples were incubated for 4 hours at $37^{\circ} \mathrm{C}$ and $5 \% \mathrm{CO}_{2}$ under dark conditions. Then, the medium was removed and $0.5 \mathrm{~mL}$ of isopropanol-HCl solution were added. Finally, the absorbance was measured at $460 \mathrm{~nm}$.

\subsection{Statistics}

Statistics Data are expressed as means-standard deviations of experiments. Statistical analysis was performed using the Statistical Package for the Social Sciences (SPSS) version 22 software (IBM). Statistical comparisons were made by analysis of variance (ANOVA). Subsequently, post hoc analyses were carried out to correct for multiple comparisons. In all of the statistical evaluations, $\mathrm{P}<0.01$ was considered as statistically significant.

\section{Results and discussion}

\subsection{Characterization results}

The synthesis strategies developed in this work are aimed to design multifunctional bioceramics with two main features. The first one is the stimulation of bone tissue regeneration to restore the skeletal integrity in bone defects. In this sense, the material should exhibit high bioactive behavior, that is, the capability to osteointegrate with the host tissue through the formation of an apatite-like phase on its surfaces. The second function is to deliver drugs on demand to treat the causal pathology of the bone defect, 
for instance bone infection or extirpated bone tumors. In order to reach these goals we have designed two different on demand drug delivery systems, depicted in Scheme 1. The first one has been designed on the basis that ATP molecules acted as capping agents. For this purpose ATP formed a covalent bond with the triamine attached to the external surface of the mesoporous material, capping the pores and inhibiting cargo release (solid S3). Conveniently, hydrolysis of ATP molecules induced by the overactivity of external ALP would uncap the mesopores, allowing cargo delivery in a controlled and selective way. The second system was designed by functionalizing the external surface of the MBG with 3-(triethoxysilyl)propyl isocyanate (S4). The resulting solid was treated with $\varepsilon$-poly-L-lysine, to form urea bonds with the isocyanate groups attached to the external surface to yield the final material S5. $\varepsilon$-poly-L-lysine would cover the surface of the loaded solid, capping the entrance to the pores and inhibiting payload release. Otherwise, the presence of bacterial proteases would induce hydrolysis of the amide bond in $\varepsilon$-poly-L-lysine, allowing cargo release. A diagram of all the prepared solids is shown in Scheme 2.

FTIR spectroscopy was carried out after each functionalization stage, confirming the presence of the corresponding functional groups for solids S1, S2, S3, S4 and S5 (see Figure S2 in Supporting Information).

The MBG functionalization was followed by solid state NMR to confirm the successful incorporation of the different components of the gates. Figure 1 shows solid-state ${ }^{29} \mathrm{Si}$ single pulse (left) and cross-polarization (right) MAS NMR spectra for solids S1, S3 and S5. These experiments were used to evaluate the presence of the organosilanes linkers bonded to the MBG surface. In solid S1, single pulse spectrum shows three signals corresponding to $\mathrm{Q}^{\mathrm{n}}$ inorganic silica environment. The observed chemical shifts, listed in Table S1 (see Supporting Information) showed signals corresponding with $\mathrm{Q}^{4}$ 
between -109 and -112 ppm, $\mathrm{Q}^{3}$ between -100 and -103 ppm and $\mathrm{Q}^{2}$ between -91 and $94 \mathrm{ppm}$. In solid S3, the resonances at around -58 and -67 ppm represent silicon atoms in positions $(\equiv \mathrm{SiO})_{2} \mathrm{Si}(\mathrm{OH}) \mathrm{R}$ and $(\equiv \mathrm{SiO})_{3} \mathrm{SiR}$, denoted as $\mathrm{T}^{2}$ and $\mathrm{T}^{3}$, respectively, evidencing the covalent functionalization with 3-[2-(2aminoethylamino)ethylamino]propyl-trimethoxysilane. These signals are emphasized in the CP spectra pointing out that the liker is mainly located at the MBG surface. In the case of solid S5, the functionalization with 3-(triethoxysilyl) propylisocyanate seems to be lower, as SP spectra does not shown $\mathrm{T}^{\mathrm{n}}$ signals and only CP spectra evidenced the presence of this organosilane at the surface of S5 [42].

Solid-state ${ }^{31} \mathrm{P}$ single pulse MAS NMR spectra for sample $\mathbf{S} 3$ were used to evaluate the local environment of P (Figure 2). The band at $1.8 \mathrm{ppm}$ is associated with inorganic $\mathrm{PO}_{4}$ tetrahedrons within silica network. Bands at -7.2, -12.0 and -20.6 ppm are assigned to $\alpha, \beta$ and $\gamma \mathrm{P}$ atoms of the ATP molecules bounded. The higher intensity of the signal at $-7.2 \mathrm{ppm}$ is attributed to the convolution of the $\mathrm{P}_{\gamma}$ of ATP with the $\mathrm{q}^{1}$ signal corresponding to environmental P-O-Si bonds $[43,44]$.

Solid-state ${ }^{1} \mathrm{H} \rightarrow{ }^{13} \mathrm{C}$-CPMAS for solids S3 and S5 are shown in Figure 3. The spectra provide clear evidence that both solids were functionalized with their corresponding gates. Solid S3 presents characteristic bands of amino-carbon bond associated with 3[2-(2-aminoethylamino)ethylamino]propyl-trimethoxysilane between 10 and 60 ppm. Characteristic bands of ATP appear from 60 ppm and correspond with the carbons of ribose and the aromatic heterocycle. S5 shows bands associated with 3(triethoxysilyl)propylisocyanate and isocyanate carbon no reacted of S4. The spectra also show characteristic band of $\varepsilon$-poly-L-lysine as amide carbons at $170 \mathrm{ppm}$.[45] The assignations of carbons are summarized in Table S2 (Supporting Information). 
The mesoporous structure of the different solids was confirmed by TEM (Figure 4).

The characteristic channels of a mesoporous ordering matrix were observed as alternate black and white stripes, evidencing highly ordered mesoporous structures in all solids, assignable to a pseudo-hexagonal group (assessed by powder X-ray diffraction measurements, see Supplementary Material, figure S1). These results demonstrate that the loading and capping processes undergone by the MBG did not significantly affect the mesoporous ordering.

The $\mathrm{N}_{2}$ adsorption-desorption isotherms of $\mathbf{S 1}$ show a type IV curve, characteristic of mesoporous materials (Figure 5). The curve shows a H1 hysteresis loop indicating that mesopores have an opened at both ends cylinder morphology. Solids S3-Ru and S5-Ru exhibit isotherms corresponding to mesoporous materials with lower surface area and pore volume, as could be expected after loading with $\left[\mathrm{Ru}(\mathrm{bpy})_{3}\right]^{2+}$ and subsequent capping with the ATP (solid S3-Ru) or $\varepsilon$-poly-L-lysine (solid S5-Ru) based gates (see Table 2). Solid S3-Ru shows a strong decrease of textural values respect to pristine solid S1. In fact, the hysteresis loop changes to type $\mathrm{H} 2$ pointing out that the mesopore morphology shifts from open cylinders towards ink-bottle morphology as a consequence of the highly effective capping with the ATP gate. On the other hand, the textural parameters of solid S5-Ru underwent a lower decrease of textural parameters. The higher organic content of solid S3-Ru compared to S5-Ru calculated from thermogravimetry would justify the different decrease of porosity observed in these solids (see Figure S2 in Supporting Information for further details).

\subsection{Kinetic studies}

In order to investigate the gating properties of ATP and $\varepsilon$-poly-L-lysine gates, cargorelease studies were carried out with S3-Ru and S5-Ru. In a typical experiment, 2 mg of S3-Ru were suspended in water at $\mathrm{pH} 7.6$ in the presence and absence of ALP. 
Suspensions were stirred at $400 \mathrm{rpm}$ at $37^{\circ} \mathrm{C}$ for 10 hours, and at given time intervals fractions of both suspensions were taken and filtered to remove the solid. Dye released to the solution was then monitored by measuring the fluorescence of $\left[\mathrm{Ru}(\mathrm{bpy})_{3}\right]^{2+}$ at 594 $\mathrm{nm}\left(\lambda_{\mathrm{ex}} 454 \mathrm{~nm}\right) .\left[\mathrm{Ru}(\mathrm{bpy})_{3}\right]^{2+}$ delivery profiles in both, the presence and absence of ALP enzyme, are shown in Figure 6A. A negligible dye release occurs when ALP is not present. Contrarily, the concentration of $\left[\mathrm{Ru}(\mathrm{bpy})_{3}\right]^{2+}$ in the solution increased significantly in the presence of the enzyme. This behavior is consistent with a tight pore closure by ATP, which would be hydrolyzed in the presence of ALP, thus unblocking the entrance to the pores and allowing cargo release.

Similar release studies were also performed with the $\varepsilon$-poly-L-lysine-capped solid S5Ru but using pronase enzyme as trigger (Figure 6B). Delivery profiles in both, the presence and absence of pronase enzyme are shown in Figure 6B. A poor $\left[\mathrm{Ru}(\mathrm{bpy})_{3}\right]^{2+}$ release was observed in the absence of pronase, while a significant increase of payload delivery occurs when the enzyme is added to the medium. The differences in both release kinetics are due to the fact that two different capping systems and enzymes are being used. $\varepsilon$-poly-L-lysine is a long polymer with a number of amide bounds to be hydrolised, yielding L-lysine molecules. The action of the pronase enzyme allows a fast hydrolysis of the molecular gate and a quick leakage of the dye out of the pores. However, alkaline phosphatase is expected to hydrolyze the phosphate groups of ATP. This anion, in solid S3-Ru, is coordinated with positively charged amino moieties located onto a dense network of polyamines grafted onto the outer surface of the loaded support. For this reason, alkaline phosphatase could have some steric hindrance in the hydrolysis of the phosphate groups of ATP and, as a consequence, a slower release of the dye was observed. 
These experiments confirm that $\varepsilon$-poly-L-lysine is a suitable capping system in MBG supports, which can be hydrolysed in the presence of pronase enzyme, thus resulting in cargo release.

\subsection{In vitro bioactivity tests}

MBGs are featured by their excellent bioactive properties. Their high surface area and porosity enhance the ionic exchange with the surrounding media, thus developing an apatite-like phase onto their surface that ensures the integration with the bone under in vivo conditions. Up to date, MBGs have demonstrated to be the fastest bone grafts in developing this apatite like phase. However, the bioactivity of MBGs can be seriously harmed if the textural properties are modified. The ATP and $\varepsilon$-poly-L-lysine based gates keep the mesopores capped until the enzymatic stimuli open them. Thus, we decided to assess the bioactive behavior of solids S3 and S5. For this purpose, both solids were soaked in simulated body fluid (SBF) at $37^{\circ} \mathrm{C}$ to test their capability for nucleating and growing a newly formed apatite-like phase. The tests were carried out in absence and

presence of the corresponding enzymatic stimuli. Solid S3 does not develop any newly formed calcium phosphate layer when soaked in SBF for 3 days, in absence of stimuli. The SEM micrographs do not evidence significant changes at the MBG surface (Figure 7A and 7B). However, in the presence of ALP, i.e. when the gate is open, the MBG recovers the bioactive behavior and develops an apatite like phase after 1 day in SBF (not showed in this manuscript), which further grows after 3 days in contact with this solution (Figure 7C ). Figure 7D at high magnification shows typical morphology apatite layer. FTIR spectroscopy agrees with the SEM results in the absence and presence of ALP (Figure 7I). In the absence of ALP, the spectra show the same absorption bands before and after soaking in SBF, i.e those corresponding to the vibrations of Si-O bonds and P-O bonds in amorphous environment. On the contrary, in 
the presence of ALP, the FTIR evidence the splitting of the signal at $590-610 \mathrm{~cm}^{-1}$ in the FTIR spectra, pointing out that the newly phase observed by SEM is an apatite-like phase (Figure 7D). In addition EDS results confirm a relation $\mathrm{Ca} / \mathrm{P}=1.66$, similar relation $\mathrm{Ca} / \mathrm{P}$ to apatite phase.

On the other hand, solid S5 exhibits an excellent bioactive behavior. This solid develops a thick newly formed apatite phase in both the presence and absence of enzymatic stimuli, as evidenced by SEM (Figure 7F, 7G and 7H), EDS with a relation $\mathrm{Ca} / \mathrm{P}=1.76$ and confirmed by the doublet at 590-610 $\mathrm{cm}^{-1}$ appeared in the FTIR spectra (Figure $7 \mathbf{J})$.

The inhibition of the bioactive behavior after the incorporation of the ATP gate could be related with the significant decrease of textural properties undergone by the MBG. The functionalization strategy used for the preparation of S3 was very efficient, as could be observed by NMR and porosimetry measurements. The capping of the mesopores would impede the ionic exchange with the surrounding SBF thus avoiding the subsequent nucleation and growth of the newly formed apatite phase. In order to find out the significance of this effect, we analyzed the $\mathrm{Ca}^{2+}-\mathrm{H}^{+}$exchange of this solid during the first stages of the bioactive process (Figure S3). The $\mathrm{Ca}^{2+}$ release from solid S3 to the SBF is significantly decreased compared with MBG without capping. $\mathrm{Ca}^{2+}$ release is the first reaction required to initiate the bioactive process [46,47]. The inhibition of this stage seriously hiders the rest of the reactions that lead to the formation of an apatitelike phase similar to the mineral component of the bone. Another possible explanation could be the entrapping of $\mathrm{Ca}^{2+}$ by the phosphate groups of the ATP gate. It has been highly demonstrated that the affinity of phosphate groups for $\mathrm{Ca}^{2+}$ can inhibit its release (and consequently the bioactivity) by forming $\mathrm{CaP}$ nanoclusters that inhibit the $\mathrm{Ca}^{2+}$ dissolution. The CaP clusters formation has been widely studied within the walls of the 
MBGs [48-51] and we hypothesize that the $\mathrm{Ca}^{2+}$ entrapment by phosphates could also occurs at the MBG surface due to the ATP presence. Independently of the mechanism that inhibits the bioactivity of S3, ALP open the ATP based gate and the MBG recovers its bioactivity.

\subsection{Biological assessment of the gated materials}

In order to test the proper working of the gatting scafold of S3-Dox before the stuies in biologial environments, controlled release of Dox in the absence and in the presecen of alkaline phosphatase were carried our. As could be seen in Figure 8, in the absence of enzyme negligible drug release was observed. However, when alkaline phosphatase was added a marked Dox release was obserbed ascribed to the hydrolysis of ATP with sugsequent pore opening. Then, the antitumoral activity of S3-Dox was studied with HOS cell cultures. HOS viability was measured in the presence of S3 and S3-Dox under absence and presence of ALP (Figure 9). The cells in contact with S3 show a proliferative behaviour very similar to those cultured on the polystyrene control. However, the cells cultured with S3-Dox clearly underwent a cytotoxic effect. In the absence of ALP, the HOS proliferation is significantly hampered after 24 hours respect to the control. This result can be due to the small amount of doxorubicin released, even without the addition of ALP. It must be taken into account that HOS cells produce ALP by themselves and could facilitate the partial opening of the ATP gates. However, 48 hours later HOS proliferate indicanting that the doxorubicin released is not enough to inhibit the growth of this tumoral cells. On the contrary, in the presence of ALP, HOS cannot proliferate after 48 hours of culture showing a significant viability decrease as a consequence of the released doxorubicin. (The complete studyat $144 \mathrm{~h}$ is described in the supplementary information in section 5). 
In a second scenario, the MBG solid capped with $\varepsilon$-poly-L-lysine was loaded with the antibiotic levofloxacin (solid S5-Levo) and the selective delivery of the antibiotic in the presence of bacteria was evaluated. For this study Escherichia coli, DH5 $\alpha$ strain, was used as model bacteria. In a first step, the amount of cargo released from S5-Levo was determined in the presence and in the absence of bacteria. As shown in Figure 10, a limited payload delivery (less than 20\% after 25 hours) was found in the absence of $E$. coli, whereas a remarkable cargo delivery was found when the bacteria were present. From these studies it was found that maximum amount of levofloxacin released from S5-Levo was of $0.322 \mathrm{ng}$ of levofloxacin per mg of solid. Moreover, it was also observed that delivery of antibiotic levofloxacin was concomitant with a reduction of bacteria viability (vide infra).

The antimicrobial activity of S5-Levo was studied more in detail by carrying clonogenic cell-viability assays in which $E$. coli bacteria were treated with different concentrations of S5-Levo at $\mathrm{pH}$ 7.6. A negative assay with no bacteria was also carried out and used as control to quantify cell growth. In a typical experiment, bacteria $\left(10^{4}\right.$ cells $\cdot \mathrm{mL}^{-1}$ ) were incubated for 5 minutes in the presence of S5-Levo, and then seeded in petri plates. Seeded plates were incubated at $37^{\circ} \mathrm{C}$ for 24 hours and then colony formation units (CFU) were quantified. Figure 11 shows \%CFU versus the maximum amount of levofloxacin that can be released from S5-Levo (i.e. $0.322 \mathrm{ng}$ of levofloxacin per mg of solid, vide ante). As seen in the figure, CFU values were correlated with the amount of S5-Levo added to the bacteria medium. S5-Levo showed considerable toxicity against $E$. coli, with an $\mathrm{EC}_{50}$ value of $22.26 \mathrm{ng} \cdot \mathrm{mL}^{-1}$. Additionally, to demonstrate that the antibiotic effect found for S5-Levo was due to the release of levofloxacin in the presence of E. coli, similar experiments were carried out with a MBG support also capped with $\varepsilon$-poly-L-lysine but that did not contain levofloxacin 
(solid S5). This solid showed negligible toxicity against bacteria (data not shown), strongly suggesting that the delivery of antibiotic levofloxacin from S5-Levo was the responsible for bacterial death. Moreover, in a further experiment the toxicity of solid S5-Levo was compared to that of free levofloxacin and free $\varepsilon$-poly-L-lysine. As depicted in Figure 11, levofloxacin and $\varepsilon$-poly-L-lysine showed similar toxicity to $E$. coli with $\mathrm{EC}_{50}$ values of $151.99 \mathrm{ng} \cdot \mathrm{mL}^{-1}$ and $131.37 \mathrm{ng} \cdot \mathrm{mL}^{-1}$, respectively. This is a remarkable result that indicated that levofloxacin is seven-fold more toxic when entrapped in S5-Levo than when free.

The toxic effect observed for S5-Levo described above against $E$. coli can be understood keeping in mind that $\varepsilon$-poly-L-lysine molecules can be hydrolyzed by proteolytic enzymes excreted by the bacteria [52] which would result in levofloxacin delivery. In order to demonstrate that protease activity of E. coli was the responsible of the release of levofloxacin via degradation of the $\varepsilon$-poly-L-lysine cap, parallel experiments were performed with solid S3-Levo. This is a MBG support loaded with the antibiotic levofloxacin and capped with the ATP gate-like ensemble described above. As the ATP gate cannot be degraded by proteases, it was expected that this solid would not be toxic for E. coli. The antimicrobial activity of solid S3-Levo was studied following a similar protocol to that described above for S5-Levo. However, in this case, no remarkable toxicity to E. coli was found after treating bacteria with S3-Levo and no levofloxacin delivery was observed.

\section{Conclusions}

In conclusion, it has been demonstrated that MBGs can be functionalized and implemented with tailored molecular gates, allowing a new application as controlled delivery device. Specifically, two different solids were prepared from a MBG selected as an inorganic support. One portion was functionalized with a triamine and capped 
with ATP, whereas the other portion was functionalized with isocyanates and capped with $\varepsilon$-poly-L-lysine. Solids following each step were correspondingly characterized, finding evidence of their correct functionalization with the tailored molecular gates. Both molecular gated mechanisms were proved to be opened, in the presence of the corresponding enzymes (ALP and pronase, respectively), while they remained closed in the absence of the stimuli. Moreover, we have demonstrated the different bioactive behavior in both gated solids. Whereas $\varepsilon$-poly-L-lysine-capped system allows the formation of crystalline hydroxyapatite on their surface under any scenario, the ATPcapped system requires the opening of the molecular gate to initiate the formation of the apatite phase.

Finally, the in vitro efficiency of both gated systems was validated. The ATP-capped system responds to the presence of high levels of ALP, opening the gates and releasing doxorubicin. Higher serum ALP levels are found in patients developing osteosarcoma and our ATP-capped system has demonstrated to inhibit the HOS cells proliferation under a similar scenario. Regarding the $\varepsilon$-poly-L-lysine-capped system, we demonstrated that the presence of E.coli bacteria was also able to hydrolyze the $\varepsilon$-polyL-lysine gate, allowing levofloxacin release. Therefore, we also tested the cytotoxic effect of the solid, and demonstrated that equitoxic concentration of the levofloxacin contained in the solid was more effective against bacteria than free levofloxacin and free $\varepsilon$-poly-L-lysine. We also proved that the capped solid performed no cellular damage when there was no levofloxacin inside the pores, which evidences that the cytotoxicity is totally caused by the released levofloxacin.

Thus, we have envisioned here a new approach to mesoporous gated materials, which is expected to set up innovative pathways to the treatment of bone diseases.

\section{Acknowledgements}


The authors thank the Spanish Government for projects MAT2015-64139-C04-01-R, MAT-2013-43299-R, MAT2015-64831-R (MINECO/FEDER) and for project CSO2010-11384-E (Agening-MICINN). Also, Generalitat Valenciana (project PROMETEOII/2014/047) and CIBER-BBN (project SPRING) are acknowledged for their support. L. Polo thanks Universitat Politècnica de València for her FPI grant. N. Gómez-Cerezo is greatly indebted to Ministerio de Ciencia e Innovación for her predoctoral fellowship. The authors also wish to thank the staff of the ICTS Centro Nacional de Microscopía Electrónica of the Universidad Complutense de Madrid (Spain) for the assistance in the scanning electron microscopy. 


\section{References}

[1] D. Arcos, M. Vallet-Regí, Sol-gel silica-based biomaterials and bone tissue regeneration, Acta Biomater. 6 (2010) 2874-2888.

[2] C. Wu, J. Chang, Mesoporous bioactive glasses: structure characteristics, drug/growth factor delivery and bone regeneration application, Interface Focus. 2 (2012) 292-306.

[3] L.L. Hench, J.M. Polak, Third-generation biomedical materials., Science. 295 (2002) 1014-1017.

[4] I. Izquierdo-Barba, D. Arcos, Y. Sakamoto, O. Terasaki, A. López-Noriega, M. Vallet-Regí, High-performance mesoporous bioceramics mimicking bone mineralization, Chem. Mater. 20 (2008) 3191-3198.

[5] X. Yan, C. Yu, X. Zhou, J. Tang, D. Zhao, Highly ordered mesoporous bioactive glasses with superior in vitro bone-forming bioactivities, Angew. Chemie - Int. Ed. 43 (2004) 5980-5984.

[6] M. Vallet-Regí, F. Balas, D. Arcos, Mesoporous materials for drug delivery, Angew Chem Int Ed Engl. 46 (2007) 7548-7558.

[7] F. Balas, M. Manzano, P. Horcajada, M. Vallet-Regí, Confinement and controlled release of bisphosphonates on ordered mesoporous silica-based materials, J. Am. Chem. Soc. 128 (2006) 8116-8117.

[8] D.P. Ferris, Y.L. Zhao, N.M. Khashab, H.A. Khatib, J.F. Stoddart, J.I. Zink, Light-operated mechanized nanoparticles, J. Am. Chem. Soc. 131 (2009) 16861688.

[9] C. Wu, J. Chang, Multifunctional mesoporous bioactive glasses for effective delivery of therapeutic ions and drug/growth factors, J. Control. Release. 193 (2014) 282-295.

[10] A. López-Noriega, D. Arcos, M. Vallet-Regí, Functionalizing mesoporous bioglasses for long-term anti-osteoporotic drug delivery, Chem. - A Eur. J. 16 (2010) 10879-10886.

[11] E. Aznar, R. Martínez-Máñez, F. Sancenón, Controlled release using mesoporous materials containing gate-like scaffoldings., Expert Opin. Drug Deliv. 6 (2009) 643-55.

[12] C. Giménez, C. de la Torre, M. Gorbe, E. Aznar, F. Sancenón, J.R. Murguía, R. Martínez-Máñez, M.D. Marcos, P. Amorós, Gated mesoporous silica nanoparticles for the controlled delivery of drugs in cancer cells., Langmuir. 31 (2015) 3753-62.

[13] E. Aznar, M. Oroval, L. Pascual, J.R. Murguía, R. Martínez-Máñez, F. Sancenón, Gated Materials for On-Command Release of Guest Molecules, Chem. Rev. 116 (2016) 561-718.

[14] C. Coll, A. Bernardos, R. Martínez-Máñez, F. Sancenón, Gated silica mesoporous supports for controlled release and signaling applications, Acc. 
Chem. Res. 46 (2013) 339-349.

[15] M. Manzano, M. Vallet-Regí, New developments in ordered mesoporous materials for drug delivery, J. Mater. Chem. 20 (2010) 5593-5604.

[16] E. Aznar, R. Villalonga, C. Giménez, F. Sancenón, M.D. Marcos, R. MartínezMáñez, P. Díez, J.M. Pingarrón, P. Amorós, Glucose-triggered release using enzyme-gated mesoporous silica nanoparticles., Chem. Commun. (Camb). 49 (2013) 6391-6393.

[17] X. Sun, Y. Zhao, V.S.Y. Lin, I.I. Slowing, B.G. Trewyn, Luciferase and luciferin co-immobilized mesoporous silica nanoparticle materials for intracellular biocatalysis, J. Am. Chem. Soc. 133 (2011) 18554-18557.

[18] R. Liu, X. Zhao, T. Wu, P. Feng, Tunable redox-responsive hybrid nanogated ensembles, J. Am. Chem. Soc. 130 (2008) 14418-14419.

[19] R. Liu, Y. Zhang, X. Zhao, A. Agarwal, L.J. Mueller, P. Feng, pH-responsive nanogated ensemble based on gold-capped mesoporous silica through an acidlabile acetal linker, J. Am. Chem. Soc. 132 (2010) 1500-1501.

[20] A. Bernardos, E. Aznar, C. Coll, R. Martínez-Máñez, J.M. Barat, M.D. Marcos, F. Sancenón, A. Benito, J. Soto, Controlled release of vitamin B2 using mesoporous materials functionalized with amine-bearing gate-like scaffoldings, J. Control. Release. 131 (2008) 181-189.

[21] N. Mas, I. Galiana, S. Hurtado, L. Mondragón, A. Bernardos, F. Sancenón, M.D. Marcos, P. Amorós, N. Abril-Utrillas, R. Martínez-Máñez, J.R. Murguía, Enhanced antifungal efficacy of tebuconazole using gated $\mathrm{pH}$-driven mesoporous nanoparticles, Int. J. Nanomedicine. 9 (2014) 2597-2606.

[22] J.L. Paris, M.V. Cabanas, M. Manzano, M. Vallet-Regí, Polymer-Grafted Mesoporous Silica Nanoparticles as Ultrasound-Responsive Drug Carriers, ACS Nano. 9 (2015) 11023-11033.

[23] N.K. Mal, M. Fujiwara, Y. Tanaka, T. Taguchi, M. Matsukata, Photo-switched storage and release of guest molecules in the pore void of coumarin-modified MCM-41, Chem. Mater. 15 (2003) 3385-3394.

[24] D. Tarn, D.P. Ferris, J.C. Barnes, M.W. Ambrogio, J.F. Stoddart, J.I. Zink, A reversible light-operated nanovalve on mesoporous silica nanoparticles, Nanoscale. 6 (2014) 3335-3343.

[25] A. Schlossbauer, S. Warncke, P.M.E. Gramlich, J. Kecht, A. Manetto, T. Carell, T. Bein, A programmable DNA-based molecular valve for colloidal mesoporous silica., Angew. Chem. Int. Ed. Engl. 49 (2010) 4734-7.

[26] Z. Yu, N. Li, P. Zheng, W. Pan, B. Tang, Temperature-responsive DNA-gated nanocarriers for intracellular controlled release., Chem. Commun. (Camb). 50 (2014) 3494-7.

[27] L. Mondragón, N. Mas, V. Ferragud, C. de la Torre, A. Agostini, R. MartínezMáñez, F. Sancenón, P. Amorós, E. Pérez-Payá, M. Orzáez, Enzyme-responsive intracellular-controlled release using silica mesoporous nanoparticles capped 
with E-poly-L-lysine, Chemistry. 20 (2014) 5271-5281.

[28] Z. Zhang, D. Balogh, F. Wang, I. Willner, Smart mesoporous $\mathrm{SiO}_{2}$ nanoparticles for the DNAzyme-induced multiplexed release of substrates, J. Am. Chem. Soc. 135 (2013) 1934-1940.

[29] Z. Zhang, F. Wang, D. Balogh, I. Willner, pH-controlled release of substrates from mesoporous $\mathrm{SiO} 2$ nanoparticles gated by metal ion-dependent DNAzymes, J. Mater. Chem. B. 2 (2014) 4449-4455.

[30] Y.-L. Sun, Y. Zhou, Q.-L. Li, Y.-W. Yang, Enzyme-responsive supramolecular nanovalves crafted by mesoporous silica nanoparticles and cholinesulfonatocalix[4]arene [2]pseudorotaxanes for controlled cargo release., Chem. Commun. (Camb). 49 (2013) 9033-5.

[31] F. Porta, G.E.M. Lamers, J. Morrhayim, A. Chatzopoulou, M. Schaaf, H. den Dulk, C. Backendorf, J.I. Zink, A. Kros, Folic Acid-Modified Mesoporous Silica Nanoparticles for Cellular and Nuclear Targeted Drug Delivery, Adv. Healthc. Mater. 2 (2013) 281-286.

[32] C. de la Torre, I. Casanova, G. Acosta, C. Coll, M.J. Moreno, F. Albericio, E. Aznar, R. Mangues, M. Royo, F. Sancenón, R. Martínez-Máñez, Gated mesoporous silica nanoparticles using a double-role circular peptide for the controlled and target-preferential release of doxorubicin in CXCR4-expresing lymphoma cells, Adv. Funct. Mater. (2014).

[33] C. Coll, L. Mondragón, R. Martínez-Máñez, F. Sancenón, M.D. Marcos, J. Soto, P. Amorós, E. Pérez-Payá, Enzyme-mediated controlled release systems by anchoring peptide sequences on mesoporous silica supports, Angew. Chemie Int. Ed. 50 (2011) 2138-2140.

[34] A. Ultimo, C. Giménez, P. Bartovsky, E. Aznar, F. Sancenón, M.D. Marcos, P. Amorós, A.R. Bernardo, R. Martínez-Máñez, A.M. Jiménez-Lara, J.R. Murguía, Targeting Innate Immunity with dsRNA-Conjugated Mesoporous Silica Nanoparticles Promotes Antitumor Effects on Breast Cancer Cells., Chemistry. 22 (2016) 1582-6.

[35] B.G. Trewyn, S. Giri, I.I. Slowing, V.S.-Y. Lin, Mesoporous silica nanoparticle based controlled release, drug delivery, and biosensor systems., Chem. Commun. (Camb). (2007) 3236-3245.

[36] H.M. Lin, W.K. Wang, P.A. Hsiung, S.G. Shyu, Light-sensitive intelligent drug delivery systems of coumarin-modified mesoporous bioactive glass, Acta Biomater. 6 (2010) 3256-3263.

[37] N. Mas, D. Arcos, L. Polo, E. Aznar, S. Sánchez-Salcedo, F. Sancenón, A. García, M.D. Marcos, A. Baeza, M. Vallet-Regí, R. Martínez-Máñez, Towards the development of smart 3D "gated scaffolds” for on-command delivery, Small. 10 (2014) 4859-4864.

[38] N. Gómez-Cerezo, I. Izquierdo-Barba, D. Arcos, M. Vallet-Regí, Tailoring the biological response of mesoporous bioactive materials, J. Mater. Chem. B. 3 (2015) 3810-3819. 
[39] M.P. Nandakumar, A. Cheung, M.R. Marten, Proteomic analysis of extracellular proteins from Escherichia coli W3110., J. Proteome Res. 5 (2006) 1155-1161.

[40] K. Haddadi, F. Moussaoui, I. Hebia, F. Laurent, Y. Le Roux, E. coli proteolytic activity in milk and casein breakdown., Reprod. Nutr. Dev. 45 485-96.

[41] G. Bacci, A. Longhi, S. Ferrari, S. Lari, M. Manfrini, D. Donati, C. Forni, M. Versari, Prognostic significance of serum alkaline phosphatase in osteosarcoma of the extremity treated with neoadjuvant chemotherapy: Recent experience at Rizzoli Institute, Oncol. Rep. 9 (2002) 171-175.

[42] I. Trenda, Á. Szegedi, K. Yoncheva, P. Shestakova, J. Mihály, A. Risti, S. Konstantinov, M. Popova, European Journal of Pharmaceutical Sciences A pH dependent delivery of mesalazine from polymer coated and drug-loaded SBA-16 systems, 81 (2016) 75-81.

[43] M.J. Potrzebowski, J. Gajda, W. Ciesielski, I.M. Montesinos, Distance measurements in disodium ATP hydrates by means of 31P double quantum twodimensional solid-state NMR spectroscopy, J. Magn. Reson. 179 (2006) 173181.

[44] U.A. Hellmich, W. Haase, S. Velamakanni, H.W. van Veen, C. Glaubitz, Caught in the Act: ATP hydrolysis of an ABC-multidrug transporter followed by realtime magic angle spinning NMR, FEBS Lett. 582 (2008) 3557-3562.

[45] S. Huh, J.W. Wiench, J. Yoo, M. Pruski, V.S. Lin, Organic Functionalization and Morphology Control of Mesoporous Silicas via a Co-Condensation Synthesis Method, (2003) 4247-4256.

[46] T. Kokubo, H. Kushitani, S. Sakka, T. Kitsugi, T. Yamamuro, Solutions able to reproduce in vivo surface-structure changes in bioactive glass-ceramic A-W3, J. Biomed. Mater. Res. 24 (1990) 721-734.

[47] L.L. Hench, Biomaterials, Science 208 (1980) 826-831.

[48] C. Turdean-Ionescu, B. Stevensson, I. Izquierdo-Barba, A. García, D. Arcos, M. Vallet-Regí, M. Edén, Surface Reactions of Mesoporous Bioactive Glasses Monitored by Solid-State NMR: Concentration Effects in Simulated Body Fluid, J. Phys. Chem. C. 120 (2016) 4961-4974.

[49] R. Mathew, C. Turdean-Ionescu, B. Stevensson, I. Izquierdo-Barba, A. García, D. Arcos, M. Vallet-Regí, M. Edén, Direct probing of the phosphate-ion distribution in bioactive silicate glasses by solid-state NMR: Evidence for transitions between random/clustered scenarios, Chem. Mater. 25 (2013) 18771885.

[50] A. García, M. Cicuéndez, I. Izquierdo-Barba, D. Arcos, M. Vallet-Regí, Essential Role of Calcium Phosphate Heterogeneities in 2D-Hexagonal and 3D-Cubic SiO 2 - CaO-P 2 O 5 Mesoporous Bioactive Glasses, Chem. Mater. 21 (2009) 54745484.

[51] E. Leonova, I. Izquierdo-Barba, D. Arcos, A. López-Noriega, N. Hedin, M. Vallet-Regí, M. Edén, Multinuclear Solid-State NMR Studies of Ordered Mesoporous Bioactive Glasses, J. Phys. Chem. C. 112 (2008) 5552-5562. 
[52] T. Yoshida, T. Nagasawa, epsilon-Poly-L-lysine: microbial production, biodegradation and application potential., Appl. Microbiol. Biotechnol. 62 (2003) 21-6. 


\section{Figure caption}

Scheme 1. Schematic representation of the ATP (A) and $\varepsilon$-poly-L-lysine (B) molecular gates.

Scheme 2. Flow diagram showing the preparation and name of the solids used in this paper.

Figure 1. Solid-state ${ }^{29}$ Si single-pulse (left) and cross-polarization (right) MASNMR spectra of S1, S3 and S5 solids. The areas for the $\mathrm{Q}^{\mathrm{n}}$ units were calculated by Gaussian line-shape deconvolutions (their relative populations are expressed as percentages).

Figure 2. Solid-state ${ }^{31} \mathrm{P}$ single-pulse MASNMR spectra (with their respective phosphorus environments shown at the top) of solid S3.

Figure 3. Solid-state ${ }^{13} \mathrm{C}$ single-pulse MASNMR spectra (with their respective carbon environments shown at the top) of solids S3 and S5. * Signal assigned to unreacted isocyanate groups.

Figure 4. Representative TEM images of solids S1, S4 and S5.

Figure 5. $\mathrm{N}_{2}$ adsorption-desorption isotherms for solids S1, S3-Ru and S5-Ru.

Figure 6. Kinetic dye release studies carried out at $37^{\circ} \mathrm{C}$ A) MBG capped with ATP (S3-Ru), B) MBG capped with ع-poly-L-lysine (S5-Ru).

Figure 7. SEM micrographs of solid S3 in absence of stimuli, A) before soaking, B) when is soaked in SBF for 24 hours and C) 72 hours. SEM micrographs of solid S3 in presence of stimuli, D) when is soaked in SBF for 24 hours and E) 72 hours. SEM micrographs of solid S5 in absence of stimuli, G) before soaking, H) when is soaked in SBF for 24 hours and I) 72 hours. SEM micrographs of solid S5 in presence of stimuli, J) when soaked in SBF for 24 hours and K) 72 hours. FTIR spectra for solids F) S3 and L) S5. 
Figure 8. Kinetic doxorubicin release studies carried out at $37^{\circ} \mathrm{C}$ with S3-Dox in the presence and in the absence of ALP.

Figure 9. HOS viability in contact with S3 and S3-Dox solids in the absence and presence of ALP after 6, 24 and $48 \mathrm{~h} \mathrm{n}=3$ control Significant differences from 48 hours: ${ }^{*} \mathrm{p}<0.05,{ }^{*} \mathrm{p}<0.01$. Statistical significance: ${ }^{*} \mathrm{p}<0.05,{ }^{* *} \mathrm{p}<0.01$

Figure 10. Kinetic levofloxacin release studies carried out at $37^{\circ} \mathrm{C}$ with S5-Levo in the presence and in the absence of E. coli.

Figure 11. Free levofloxacin $(\bullet)$, free $\varepsilon$-poly-L-lysine $(\circ)$, equitoxic concentration of levofloxacin in S5-Levo (-) and equitoxic concentration of levofloxacin in S3-Levo ( $\mathbf{\Delta}$ ) versus \%CFU. 